\title{
Haïti première république noire des lettres
}

\section{Michael Dash}

\section{(2) OpenEdition}

\section{Journals}

Édition électronique

URL : http://journals.openedition.org/actesbranly/480

DOI : $10.4000 /$ actesbranly.480

ISSN : 2105-2735

\section{Éditeur}

Musée du quai Branly Jacques Chirac

\section{Référence électronique}

Michael Dash, "Haïti première république noire des lettres », Les actes de colloques du musée du quai Branly Jacques Chirac [En ligne], 3 | 2011, mis en ligne le 21 avril 2011, consulté le 07 septembre 2020. URL : http://journals.openedition.org/actesbranly/480; DOI : https://doi.org/10.4000/actesbranly.480

Ce document a été généré automatiquement le 7 septembre 2020

(c) Tous droits réservés 


\title{
Haïti première république noire des lettres
}

\author{
Michael Dash
}

1 J'ai divisé la communication en trois partie, la première partie s'appelle « La nouvelle terre-mère ", la deuxième partie : « une nouvelle littérature monde » et la troisième : « Emma Bovary, c'est nous ».

\section{La nouvelle terre-mère}

Dans l'étude historique Les Jacobins noirs, publiée en 1938, CLR James a montré que la centralité de la Révolution haïtienne était due au fait que, pour la première fois, dans le Nouveau Monde, des Africains avaient pris conscience qu'ils formaient un peuple moderne. Ils avaient créé une nouvelle vision de la modernité qui plus tard deviendrait un élément essentiel du patrimoine du monde noir.

Dans cet ouvrage, James contemplait aussi les conséquences intellectuelles de la révolution de Saint- Domingue. Grâce à son existence, les origines intellectuelles du monde noir n'étaient à chercher ni dans les anciennes métropoles coloniales, ni dans l'Afrique ancestrale. Haïti n'était pas la fille aînée de l'Afrique mais elle était devenue la nouvelle «terre-mère » idéologique, comme l'a affirmé Edouard Glissant, le lieu où la lutte anti-coloniale a produit une conscience collective, une nouvelle façon de penser la question raciale, voire même, une nouvelle façon de concevoir l'identité nationale. Pour ces raisons, dans sa pièce Monsieur Toussaint, Glissant envisage un espace théâtral où il n'y a pas de restauration du passé historique mais qui est un lieu d'échanges et de résonances. Glissant imagine que la cellule de Toussaint, dans sa prison du Jura, est un lieu de rencontre qui ouvre le passé haïtien vers un avenir déterritorialisé. Voir la révolution haïtienne comme un lieu de passage ouvert à tous, libère donc le passé haïtien des deux cents ans de solitude imposés par l'Occident, et démontre que l'héritage révolutionnaire d'Haïti n'est limité ni à un seul pays, ni à un seul peuple. De ce fait, dans Monsieur Toussaint, la révolution haïtienne a plusieurs horizons - des horizons caribéens et hémisphériques autant que des horizons africains et européens. 
Bien que la pièce se déroule dans la cellule du fort de Joux, Toussaint est en dialogue constant aussi bien avec des personnages du passé qu'avec ses contemporains. Dépaysement de l'histoire, déplacement physique et métaphorique du héros, sont les outils qu'utilise Glissant pour mettre l'accent sur la portée internationale de l'événement révolutionnaire haïtien.

De plusieurs points de vue, cette terre matrice a créé une révolution originale qui dépassait autant les limites idéologiques et intellectuelles que les frontières de l'imaginaire. Ce qui permettait aux révolutionnaires de Saint-Domingue d'exécuter une œuvre originale selon l'historien haïtien Michel-Rolph Trouillot c'était le fait que «the Haitian revolutionaries were not overly restricted by previous ideological limits set by professional intellectuals in the colony or elsewhere, they could break new ground and indeed did so repeatedly ». James a abordé cette question d'une façon assez énigmatique dans Les Jacobins noirs. Il a suggéré que les esclaves de Saint-Domingue avaient saisi l'essentiel du projet révolutionnaire français en associant universalisme et droits de l'homme. Ainsi la nature humaine devenait transculturelle et transhistorique, par delà toutes particularités régionales ou ethniques. Selon James les esclaves avaient compris le principe d'universalité des droits de l'homme et l'avaient appliqué à leur propre situation. «They had heard of the revolution and had construed it in their own image : the white slaves in France had risen, and killed their masters, and were now enjoying the fruits of the earth. It was gravely inaccurate in fact, but they had caught the spirit of the thing. " Ce que James appelle "l'esprit de la chose » est l'élément essentiel du radicalisme de la révolution de Saint-Domingue.

5 L'application radicale de l'esprit révolutionnaire, l'intraduisible « spirit of the thing » a permis qu'on accorde à tous les citoyens noirs l'égalité de droits. En d'autres termes, elle a rendu possible la réalisation d'un projet politique qui au départ n'était présent ni dans la révolution française, ni dans la révolution américaine toutes deux plus conservatrices que la révolution haïtienne.

Mais les analyses de James sont restées lettre morte. Une année à peine après la parution des Jacobins noirs, Aimé Césaire publiait le Cahier d'un retour au pays natal, poème dans lequel il faisait de la révolution haïtienne un symbole de la rédemption raciale et dépeignait Toussaint sous les traits d'un héros restaurant la dignité du peuple noir. Le poème unit le projet révolutionnaire haïtien à l'idéologie de la négritude dans une formule célèbre : « Haïti où la négritude se mit debout pour la première fois. » La vision que s'est faite Césaire de la révolution haïtienne est celle d'une lutte héroïque menée par un peuple isolé qui, « seul contre tous, s'est libéré de l'esclavage ». Césaire a beaucoup insisté sur les dimensions raciales et épiques de la révolte des esclaves de Saint-Domingue. Par exemple, dans le poème Le verbe marronner, où il invitait René Depestre à marronner le parti communiste et les conseils poétiques de Louis Aragon, il demandait au poète haïtien de ne pas oublier le «chant dément de Boukmann accouchant (son) pays aux forceps de l'orage». Si on suit Césaire, c'est en fait Boukmann qui a donné naissance à la nation haïtienne dans le Bois Caïman. Le poète martiniquais privilégie donc les thèmes du marronnage, de l'affirmation raciale et $\mathrm{du}$ sang. Il voit dans Boukmann un nègre fondamental et fondateur dont le «chant dément » devrait guider Depestre.

7 La popularité du Cahier ne doit cependant pas nous faire croire que l'interprétation césairienne de la révolution haïtienne a triomphé. De fait, du $\mathrm{XIX}^{\mathrm{e}}$ au $\mathrm{xxI}^{\mathrm{e}}$ siècle, la vision humaniste et globalisante de la révolution comme événement dépassant les 
frontières réelles et symboliques du pays où elle s'était déroulée, s'est manifestée à de nombreuses reprises dans des œuvres littéraires qui essayaient de défendre l'héritage révolutionnaire.

\section{Une première littérature-monde}

8 Si l'idée d'un universalisme révolutionnaire a été préservée dans la littérature noire, c'est d'abord grâce aux écrivains haïtiens du XIX ${ }^{e}$ siècle. La plupart des intellectuels de cette époque contestaient l'idée de la singularité haïtienne dans leurs études de la réalité culturelle et politique de leur pays. Ils avaient senti que l'idée de la nature exceptionnelle d'Haïti était la création d'un discours raciste qui cherchait à exclure Haïti de la scène mondiale. Michel-Rolph Trouillot nous rappelle que :

9 «Before the twentieth century; Haitian writers rarely if ever promoted singularity in their studies of Haitian reality. Indeed, Haitian intellectuals rightly saw the theories of Haitian exceptionalism that were spreading in Europe and North America as implicitly -and often explicitly -racist... these writers did not think that Haiti escaped the paradigms of their times. "

10 Ainsi Trouillot démontre que, dès le début, les intellectuels haïtiens, fidèles au message universel de la révolution, contestaient les processus de marginalisation dont leur pays était victime à cause de sa prétendue spécificité raciale et culturelle. Dans un contexte politique mondial ou les différentes puissances coloniales se partageaient le monde, cette position était profondément radicale.

11 Pour illustrer cette position intellectuelle, nous pouvons nous tourner vers Antênor Firmin qui, à la fin du XIX ${ }^{e}$ siècle, s'appuyait sur l'idéal humaniste pour rejeter les thèses de Joseph-Arthur de Gobineau sur la hiérarchie des races. Dans son monumental De l'égalité des races humaines, il concluait que les êtres humains partout avaient les mêmes qualités et qu'ils étaient tous liés par « une châne invisible » qui était leur humanité commune. Depuis son exil de saint Thomas, il fustigeait la xénophobie qui avait trahi l'idéal de la modernité inauguré par la révolution et voyait dans une confédération caribéenne le seul moyen de résister au néo-colonialisme américain.

12 Plus près de nous, au $x^{e}$ siècle, la portée de l'œuvre de Jacques Roumain ne se mesure pas uniquement au succès du mythe fondateur de la nation, qu'il a créé. Son roman, Gouverneurs de la rosée, révèle aussi à quel point il était sensible à la globalisation de la culture populaire. À la différence des partisans du noirisme, Roumain ne pensait pas que l'espoir d'un changement révolutionnaire résidait dans la figure du marron enraciné dans l'arrière pays. Pour lui, cet espoir était porté par le travailleur, politiquement radicalisé que l'impérialisme américain avait poussé à la migration. Roumain était conscient que la culture paysanne de son pays n'existait plus à l'état d'isolement absolu et que les mouvements des marchandes et des spéculateurs qui liaient régulièrement la campagne aux villes, mettait en contact culture paysanne et culture urbaine.

13 On a beaucoup mis l'accent sur la manière dont le lyrisme poétique de Gouverneurs de la rosée s'associe étroitement à l'évocation mythique de l'espace haïtien. Mais on oublie qu'il y a aussi dans le texte une autre poésie née de la pluralité culturelle, en un mot, de l'hybridation. Manuel, dont le nom réunit à la fois le familier et l'insolite, le banal et le divin, est non seulement le maître des sources mais le maître des carrefours. Le voyage 
qui l'amène dans les plantations de Cuba lui apprend que l'expansionnisme américain rendait obsolète les concepts d'identité territoriale et d'espace cultuel exclusif. Ce voyage lui a permis de réinterpréter la pratique traditionnelle du coumbite ${ }^{1}$ à la lueur de son expérience de la huelga (la grève). Pour lui, il ne s'agissait plus de paysans du même clan travaillant la terre de leur voisin ou de leur frère. L'affinité de sang avait cédé à une autre réalité économique ou à celle du travailleur migrant sur la scène mondiale. Les houes, scintillant dans le soleil, des paysans prométhéens de Fonds Rouge, ont été remplacées par les poings des grévistes et le bruit du tambour, par « un NON à mille voix qui ne font qu'une et qui s'abat sur la table du patron avec le pesant d'une roche ». D'une certaine manière le paysage pastoral fait place au paysage de résistance que composent les épaules des travailleurs solidaires : «On est soudé comme les épaules des montagnes et quand la volonté de l'homme se fait haute et dure comme les montagnes il n'y a pas de force sur terre ou en enfer pour l'ébranler et la détruire ».

14 Ce qui fait la force de l'imaginaire de Roumain c'est qu'il était capable de prévoir et prédire l'impasse des recherches de l'atavisme culturel du mouvement indigéniste haïtien des années 1920. Il signifiait aux lecteurs haïtiens qu'il était profondément dangereux de vivre dans l'isolement culturel.

\section{Emma Bovary, c'est nous}

15 Comme suggéré plus tôt, c'est Edouard Glissant qui a le plus essayé de penser la révolution comme un espace ouvert, un lieu de rencontre. Comme l'avait fait remarqué Victor Segalen, l'écrivain qui a le plus influencé Glissant, l'important n'est pas de décrire un objet «mais [d']en donner un équivalent poétique ». Cette idée sera mise à l'œuvre dans Monsieur Toussaint que Glissant a décrit comme une tentative poétique d'éprouver le passé haïtien "dans son épaisseur ». Glissant a voulu résister au désir de traduire la révolution. Il n'a pas cherché à restituer le passé mais à le recomposer poétiquement comme un lieu d'échanges et de résonances.

16 La question de la représentation poétique de la réalité est d'une importance primordiale surtout maintenant où on a tendance à associer le projet d'une littératuremonde avec le retour du référent. Michel le Bris, par exemple, critique sévèrement la préoccupation textuelle et formaliste de la littérature française contemporaine et voit dans la littérature francophone un désir de se tourner vers le monde et de l'écrire directement. Édouard Glissant, qui se voit comme solitaire et solidaire dans le projet de Littérature-monde, critique indirectement une simplification dangereuse qu'il appelle la littéralité c'est-à-dire le désir de rendre le réel transparent qui se manifeste dans les propos de Le Bris. Ce qu'il propose c'est la recherche d'une poétique pour exprimer un réel qui est imprévisible et opaque.

17 La question de la représentation du réel haïtien était d'une importance primordiale au $\mathrm{XIX}^{\mathrm{e}}$ siècle. Par exemple, dans les pages du journal Le Républicain, en octobre 1836, Emile Nau a proposé le concept de métissage culturel comme un moyen de définir l'identité haïtienne. Il voyait dans la fusion des cultures africaines et européennes quelque chose qui rendait l'haïtien moins français que le blanc américain n'était anglais. Dès lors se posait le problème de la représentation d'un processus inachevé, d'un processus capable de produire un nouvel ordre de différence dans le monde. Toutes les écoles littéraires $\mathrm{du} \mathrm{XIX}^{\mathrm{e}}$ siècle ont essayé en vain de répondre à ce dilemme. Ni le romantisme, ni le réalisme, ni le symbolisme n'ont pu fournir aux Haïtiens les outils nécessaires pour 
résoudre le problème de la représentation. Les penseurs haïtiens avaient senti que ce n'était pas une simple question de dialogue avec le monde mais ils étaient dans le monde aussi. C'est une conscience de la force structurante du monde qu'ils étaient incapables de systématiser esthétiquement.

Pendant l'occupation américaine, Jean Price-Mars a tenté de figer l'identité nationale dans Ainsi parla l'oncle (1928). En essayant de promouvoir l'idéal d'une authenticité culturelle pour Haïti, il a utilisé le concept de bovarysme que Jules de Gaultier avait défini comme «le pouvoir de l'homme de se concevoir autre qu'il n'est ». Price-Mars avait utilisé cette idée pour critiquer ce qu'il voyait comme l'aliénation culturelle ou le bovarysme collectif de l'élite haïtienne. Il avait demandé aux Haïtiens de cesser de nier leur « moi collectif » et de se tourner vers le passé africain. Par la suite, le Duvalierisme se servira précisément de l'idée de « moi collectif » pour légitimer une dictature noire et pour écraser l'élite mulâtre.

La littérature post-Duvalieriste va témoigner de son désir de rompre avec la perspective réductionniste de Price-Mars et de repenser les termes du bovarysme en refusant de stabiliser la différence haïtienne. Le dilemme qu'Emile Nau avait posé en 1836 refait donc surface. Comment représenter le réel haïtien? Pour Price-Mars une société qui se conçoit autre qu'elle n'est, est une société aliénée. Pour un écrivain contemporain comme Dany Laferrière, c'est le contraire. Il a dédié son avant-dernier roman, Je suis un écrivain japonais (2008), «à tous ceux qui voudraient être quelqu'un d'autre ». Cette dédicace semble être une réponse directe et provocante à la vision de l'identité haïtienne monolithique si chère à Price-Mars et aux noiristes de Francois Duvalier. La critique du noirisme haïtien, bien sûr, parcourt toute l'œuvre de Laferrière, mais elle est particulièrement incisive dans son roman autobiographique Pays sans chapeau (1996), qui par ailleurs est une réponse au Cahier d'un retour au pays natal de Césaire. Dans ce roman qui traite d'un retour au pays après une longue absence, le narrateur rend visite à l'ethnographe J-B Romain, un disciple de Price-Mars. Romain avoue qu'il est un homme du passé et qu'« on me demande mon avis sur des histoires qui se déroulent sous nos yeux. Il me faut du temps. Dans mon analyse d'Haïti je suis encore en Afrique, vous comprenez."

20 Le narrateur se moque également du docteur Legrand Bijou qui prétend voir dans l'arrière-pays haïtien le site d'une harmonie entre l'homme et la nature. En élaborant sa théorie d'une photosynthèse culturelle, Bijou déclare que «le créole de Bombardopolis est le plus pur d'Haïti » parce que « les habitants de Bombardopolis sont devenus des plantes. »

21 Dans le paratexte à la fin du livre, Laferrière revient directement à la question du bovarysme et de la représentation du réel haïtien. Il évoque alors l'histoire qui est à l'origine du roman. C'est celle de son voisin, Baptiste, un peintre qui créait des toiles irréelles. Lorsqu'un journaliste américain demande à Baptiste pourquoi il ne peint pas le pays réel, il lui répond qu'il n'a pas besoin de le peindre puisqu'il le rêve. Au lieu de copier la réalité, Baptiste préfère rendre poétiquement un réel qui reste énigmatique et impénétrable. Comme pour Emma Bovary, chez Baptiste, le rêve a remplacé le réel. En fin de compte, ce peintre devient le modèle de l'artiste idéal pour Laferrière. Dans son dernier roman L'Énigme du retour (2009), quand le neveu du narrateur dit à son oncle qu'il sent «une distance de plus en plus grande entre la réalité et [lui]», l'oncle lui répond : «C'est peut-être ton espace pour écrire. » 
En repensant la question du bovarysme, et en posant l'importance du rêve pour peindre le réel, Laferrière nous rappelle indirectement que le projet politique de la création de la nation haïtienne a toujours eu une dimension poétique. Il a toujours été une tentative de rêver, d'inventer un nouveau peuple, de refuser le déterminisme des origines biologiques pour affirmer une modernité ancrée dans l'histoire. La grande ambition de la littérature haïtienne a toujours été de continuer ce rêve profondément poétique et politique.

\section{NOTES}

1. Travail saisonnier effectué en commun.

\section{AUTEUR}

\section{MICHAEL DASH}

Né à Trinidad, J. Michael Dash est professeur au Département de Francais de New York University. Il est spécialiste de la littérature et de la culture d'Haïti sur lesquelles il a publié plusieurs ouvrages dont Literature and ideology in Haiti (1981), Haiti and the United States (1988) et Culture and Customs of Haiti (2001). Il est aussi l'auteur d'un livre sur Edouard Glissant dont il a traduit trois ouvrages. 